\title{
System Architecture and Data Flow in Applications for Cross Platform Communication in a Secure Network
}

\author{
Manish Kumar \\ M.Tech Research Scholar \\ Maharishi Markandeshwar University, Ambala
}

\author{
Debasis Dwibedy \\ Assistant Professor Dept of Computer Science \\ Maharishi Markandeshwar University, Ambala
}

\begin{abstract}
The interfacing online application system with an Intranet based ERP application is always a difficult task and contains so many threats in the process. The aim of this research paper is to describe the methods, technology, algorithms and implementation details of system architecture for cross platform communication of diversified applications over network. This paper consider author's base paper namely Implementation of a Novel System for Cross Platform Communication of Diversified Applications over Network. The paper describes the difficulties to transfer the data files from the Intranet server to Centralized web server with proxy server settings, the authors face.
\end{abstract}

This system is in Visual Studio 2010 development environment, using C\# programming language, ASP.NET, ADO.NET and SQL Server 2012 to develop a join system. To protect the data from attackers, the firewall restrictions should be setup. This paper focuses on the process of transfer data from SAP R/3 system to SQL server of local Intranet server which then makes the text files of the data and the data is transferred to the Web application server after the files are zipped. So the overall process contains difficulties to pass the Intranet firewall setup as well as to secure the whole process. The paper also focusses on the process of Bulk Copy Program, optimization of routing decision and secure data transmission through the help of web services. The various keys are associated with throughout the process and the final version of data would be final after transmission. The objective of this work is to describe the overall architecture for Cross Platform Communication of author's previous work.

\section{Keywords}

SOAP, Firewall, Web Services, Relational Databases, ASP.NET, SQL Server, Intranet, RFC, SAP, Internet Proxy Servers.

\section{INTRODUCTION}

The need for communication in the Internet grows as the number of devices - and with them the demand for safety, volume and speed. Interaction replaced information and instead of traditional client/server mechanisms, a more convenient and standardized way of data exchange is now needed - not only between client and server, but also between the various clients today. With Web services one can quickly implement an application for cross-platform communication. Using files the user can send data to other processes/system and receive replies, transfer data across multiple processes and systems, etc.

A web service provides a defined set of functionality on a machine processed interface [1] [6]. The web service interface is described in a formal language like Web service Description language that allows creating code to access the service thus simplifying web service consumer (client) and provider (server) development.As the authors defined in their previous paper that the system architecture consists of an Agent and Cloud. Agent is basically a local system or Virtual Machine which is used to connect to SAP server and download data to the local system [1]. The Agent communicates with SAP through SAP connector and RFC (Remote Functions Call) mechanism. The RFC are called using SAP Web Services using SOAP standard. The second web service is used to transfer downloaded data from SAP to Cloud server and this process is carried out using SOAP mechanism [1].

The web application is the most useful platform providing interactive GUI based system to interact with. The security must be maintained while data travel for this use [2].

Distributed firewall is a mechanism to enforce a network domain security policy through the use of policy language and security policy is defined centrally. This enables the identification of any member of the network policy domain. Distributed Firewalls secure the network endpoints, exactly where the hackers try to penetrate. It filters traffic from both the internal and internet network and overcome the single point of failure concept [7] [14].

Thus, by concentrating on these important factors, the authors first describes how the data would be collected from SAP and how the data will be stored in local SQL Server 2012. Secondly, the authors build a network where the text files are getting converted into zip folder and then transferred to the Cloud server. The various sections of the paper are organized as follows. In first section the authors discuss the existing work on web services and system integration techniques. In second section, the authors define the disadvantages of existing scenario in the internal intra-network for which the solutions need to be described and implemented in the paper. In third section, the authors define some topologies, terms, technologies and protocols to be used. In fourth section the authors describe the methods and algorithms used to implement the overall system. The fifth section of the paper provides the experiment results need to find out the further improvements. In the sixth section, the authors define the future research scope and finally conclusion in the next section.

\section{LITERATURE SURVEY}

In [1], the author provides the System for Cross Platform Communication of Diversified Applications over Network. The author provides the web services basics and defined the architecture of the system. The author provides an interface which uses SAP.Net Connector and Web services based on SOAP protocols for the data communication.

In [2], the author describes the web application security is the key fact in the Intranet level using HTTP. The author gives 
the overall architecture of web application and proposed some design for the security of the web based application.

In [3], the author describes the network security using proxy servers. The proxy servers works at the local intranet level and provides the wall (proxy address) to protect the internal data so as to achieve the wireless network security.

In [5], the author defines the routing system using an ASP.Net MVC application. The author takes routes, testing of routes and route entry in the routing table to analyze the system.

In [6], the author describes the working of HTTP Web services and Web servers to provide services to different application and how firewall is linked in the system for protection. The author also describes the SOAP architecture.

In [7], the author describes the protection of information systems against malicious attacks and invasions using embedded and distributed firewall. The author discuss conventional firewall system and provides the architecture of embedded firewall in Distributed security system.

In [10], the author describes the scalability of database bulk insertion with multi threading to enhance the performance of data access layer.

In [14], the author describes the issue of computer and network security and discussed the levels of security. The author also discuss elements of network security and password mechanisms by encryption and authentication.

In [15], the author describes the part of bridging the gap between Microsoft.Net framework technology and SAP R/3. The author tries to setup an environment between these two systems and defined two methods. One is using web services and other is using SAP.NET connector. They describe the creation of web services at Dot Net part of system and DDL creation of assemblies for RFC and SOAP communication using Dot Net proxy classes. The author defines the use of Visual Studio and system namespace to include the web services and the communication flow process in a way the author specifies is brief.

\section{MOTIVATION}

The existing interface system architecture have queries related to how the data will transfer from one independent system to another independent system. This leads to find the problems and ways to overcome those issues have been going as a research work. The main issue include:

- How the applications from other platforms can communicate with ERP systems?

- How Web Services are integrated into ERP?

- What additional values do Web Services bring to traditional ERP systems?

- What will be the issue of Firewall which works in the internal network for the sake of security in the organization?

- The choice of proxy server and the problem associated with the server?

- ERP systems didn't allow integration of the partners, customers and suppliers applications within the system. So how the model will be?

- What will be the parameters need to be considered while the system design?
- What will be the selection of a network system having a centralized firewall scenario or distributed firewall design?

\section{FIREWALL, INTRANET, RFC AND INTERNET PROXY SERVER}

Firewall is a device of protection a local system or network of systems from network-based security threats while affording access to the outside world via WAN`s or the Internet. It controls the incoming and outgoing network traffic based on applied set of rules. It establishes a barrier between a trusted, secure internal network and another network (e.g., the Internet) that is assumed not to be secure and trusted. The firewall is inserted between the premises network and the Internet and it aims:

- Establish a controlled link

- $\quad$ Protect the premises network from Internet-based attacks

- $\quad$ Provide a single choke point

- All traffic from inside to outside must pass through the firewall (physically blocking all access to the local network except via the firewall)

- Only authorized traffic (defined by the local security police) is allowed to pass

- $\quad$ The firewall itself is immune to penetration

\section{Local Area Network}

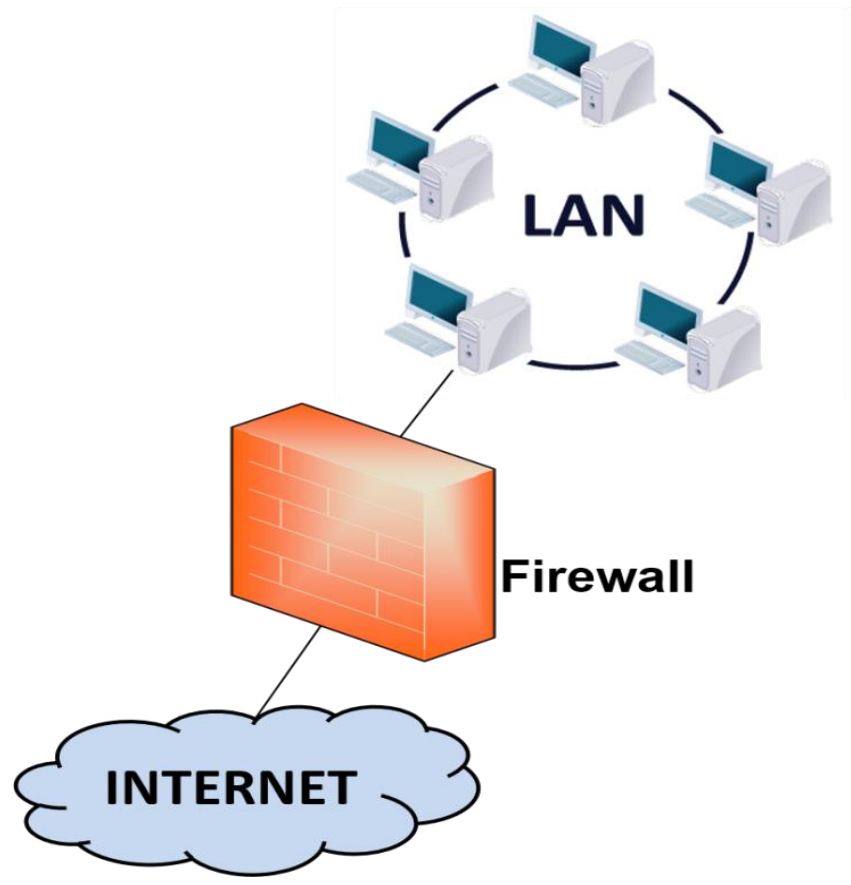

Fig 1: Firewall system architecture

The centralized firewall leaves the servers between the internal private network and the external network exposed

- Servers in this area should provide limited functionality

- No services/software they don't actually need

- $\quad$ These servers are at extreme risk 
- Vulnerable to service specific hacks - HTTP, FTP, Mail, ...

- Vulnerable to low level protocol (IP, ICMP, TCP) hacks and DoS attacks

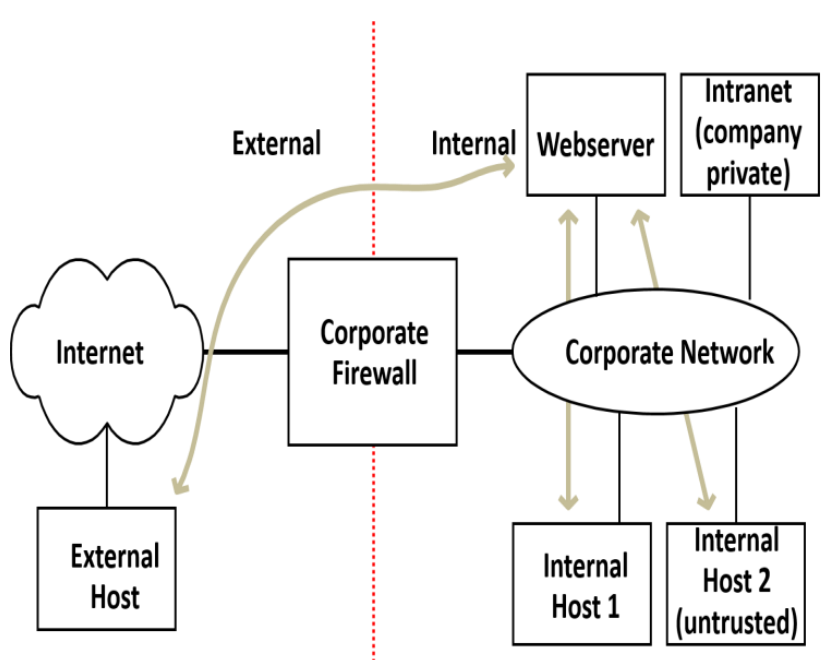

Fig 2: Centralized Firewall design in a corporate network

Intranet is an internal corporate network which uses Internet and World Wide Web standards and products that allows employees of an organization to gain access to corporate information. A network based on TCP/IP protocols (an internet) belonging to an organization, usually a corporation, accessible only by the organization's members, employees, or others with authorization. An intranet's Web sites look and act just like any other Web sites, but the firewall surrounding an intranet fends off unauthorized access. Like the Internet itself, intranets are used to share information. Secure intranets are now the fastest-growing segment of the Internet because they are much less expensive to build and manage than private networks based on proprietary protocols.

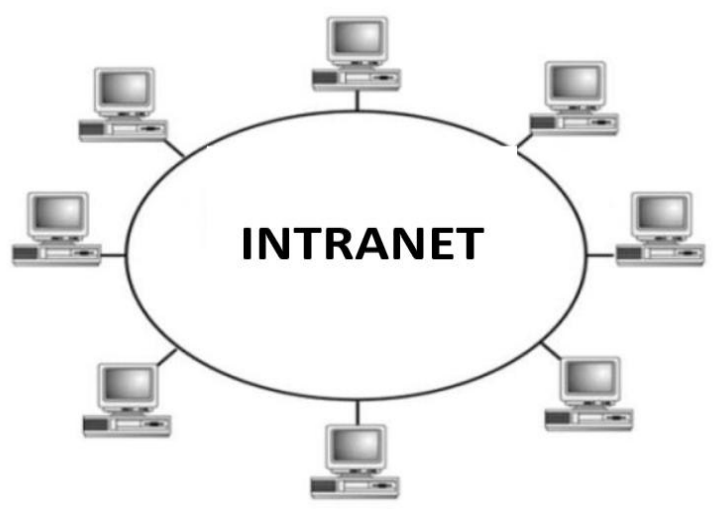

Fig 3: Intra-network of systems

The Intranet permits your organization to describe itself as a whole entity or a group where everyone knows their roles and responsibilities, and everyone is working on the improvement and health of the organization. Intranet does this by identifying and communicating missions, goals, processes, relationships, interactions, infrastructure, projects, schedules, budgets and culture on-line, in a single interface everyone uses and can add value back to. In a word, an Intranet represents your organization's "intelligence". The purpose of this intelligence is to manage each individual's desktop with minimal cost, time and effort to be more productive, more cost efficient, timelier and more competitive.

Remote Function Call is Communication between applications in different systems in the SAP environment that includes connections between SAP systems as well as between SAP systems and a no. of non-SAP systems. Remote Function Call (RFC) is the standard SAP interface for communication between SAP systems. RFC calls a function/methods to be executed in a remote system. Although an RFC associated with communication between two different systems, it can also be created for communication within a system itself.

Proxy hides internal users from the external network by hiding them behind the IP of the proxy. It prevents low level network protocols from going through the firewall eliminating some of the problems with NAT and restricts traffic to only the application level protocols being proxied. Proxy is a combination of a client and a server and internal users send requests to the server portion of the proxy which then sends the internal users requests out through its client ( keeps track of which users requested what, do redirect returned data back to appropriate user)

- Address seen by the external network is the address of the proxy

- Everything possible is done to hide the identity if the internal user (e-mail addresses in the http headers are not propagated through the proxy)

- Doesn't have to be actual part of the Firewall, any server sitting between the two networks and be used

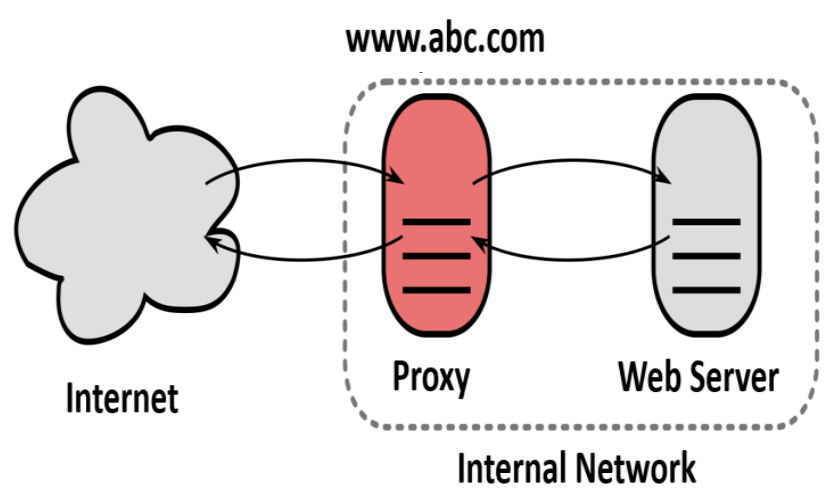

Fig 4: Proxy server setup design

\section{OVERALL SYSTEM ARCHITECTURE AND DATA FLOW}

As explained already the objectives and functions of RFC by statement, it is useful in communication between two SAP systems and SAP systems and other systems as shown below in the fig. 5. The external systems may be any kind of systems having a database (Oracle, SQL Server etc.). So the connectivity needs to be maintained while the communication. In the paper, it is considered that the SAP system and the SQL Server on the same system which is called Agent server machine.

Thus the Agent server is responsible for calling the corresponding RFCs and as per the program written within $\mathrm{RFC}$, the processing is done in the Agent application.

The detailed steps are as follow: 
- $\quad$ ASP.Net program which runs at Agent server calls the corresponding RFC with appropriate parameters.

- As per the RFC program, the SAP system generates required data which is pushed to SQL Server 2012.

- There are many filters which is used to filter the data before getting upload into the Cloud system.

- The code to call the BAPI through RFC in C\# is given below:

IRfcFunction BapiGetMasterData $=$

SapRfcRepository.CreateFunction ("/ABC/IN_");

BapiGetMasterData.SetValue ("Z_", "X");

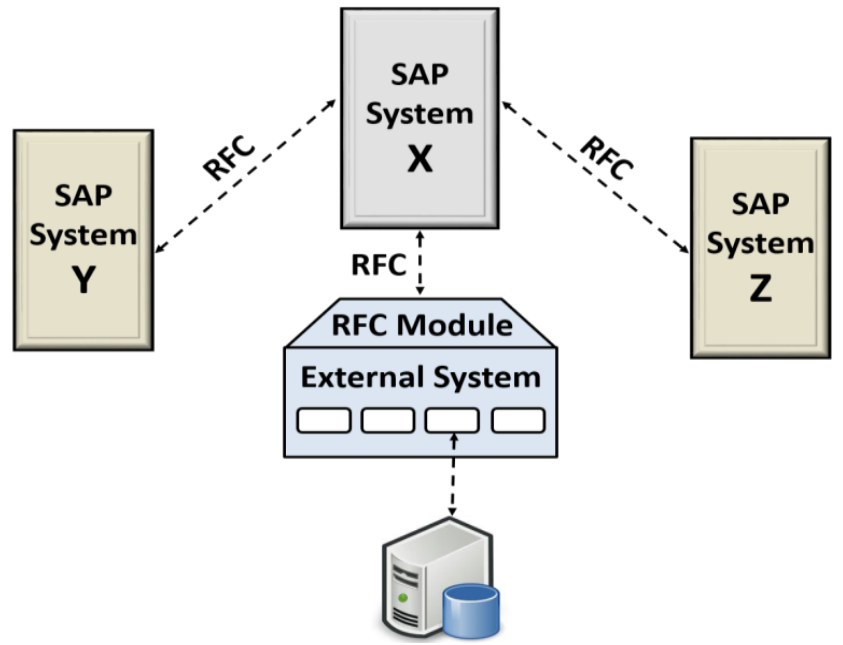

Fig 5: System communication process through RFC

Next the SQL Server prepare the text files of the data downloaded from SAP and places the files to the local server machine in the intranet which is then zipped. The process of zipping the text files is given below:

ZipFile DataZip = new ZipFile $($;

DirectoryInfo dirInfo = new

DirectoryInfo(@ConfigurationManager.AppSettings[ "BCPFolder"].ToString());

DataZip.Password =

ConfigurationManager.AppSettings["WebServicePas sword"].ToString();

\section{foreach (FileInfo $f$ in dirInfo.GetFiles())}

\{

DataZip.AddFile(f.FullName, "'");

\}

DataZip.Save(@ConfigurationManager.AppSettings[ "BCPFolder"].ToString() + "CNData.zip");

DataZip.Dispose();

The zip file is password protected from the system end for the sake of security, confidentiality, consistency, nonrepudation of data.

The main issue is now to transfer this zip folder into the Cloud web server which needs to cross the organizational internal network firewall.
The solution is to use the proxy server address and by pass the files to cloud by using the below code in $\mathrm{C \#}$ :

\section{<defaultProxy useDefaultCredentials="true"} enabled="true" >

<proxy usesystemdefault="True" proxyaddress="http://proxy-in.abc.com:8080" bypassonlocal="False"//>

\section{$</$ defaultProxy $>$}

So the issue related to uploading the zip file to cloud has been solved and the file can be uploaded after passing the firewall of the protected network. So the time can also be reduced by using the fast internet speed and proper proxy servers.

The code to upload the zip file to the web server is given below:

response = oCNS.UploadFile("CNData.zip", File.ReadAllBytes(ConfigurationManager.AppSetting s["BCPFolder"].ToString() + "CNData.zip"));

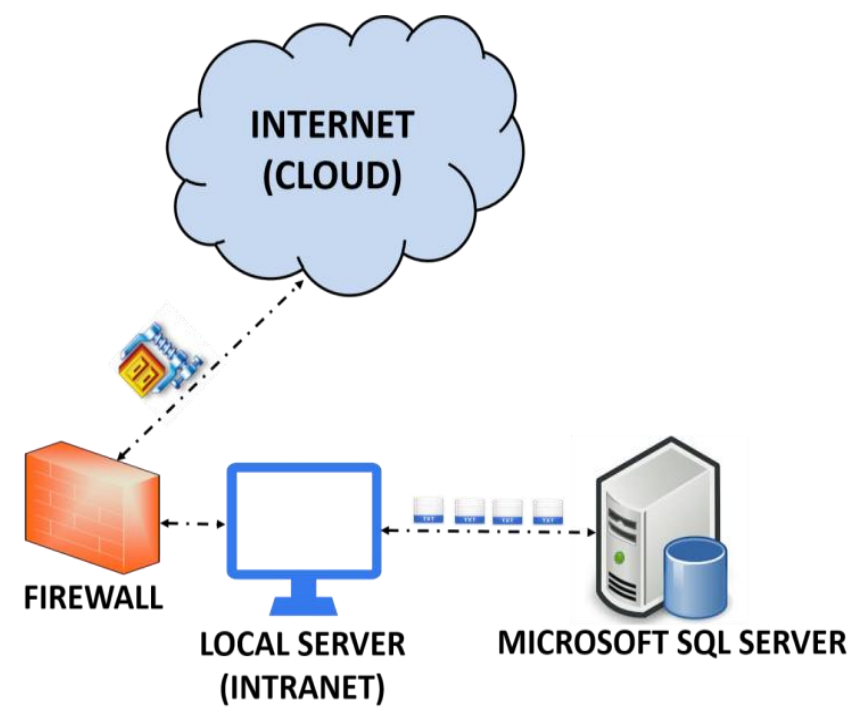

Fig 6: Processes in Agent server end

The users on the Agent system use the web browser (mostly Internet Explorer) to run the application and the corresponding data download, data upload and synchronization of the SAP data from local system to web browser takes place whenever required. So the data will have to cross the internal firewall which the authors had discussed earlier. The snapshot showing how the internal user will use the Agent is shown in fig.7 


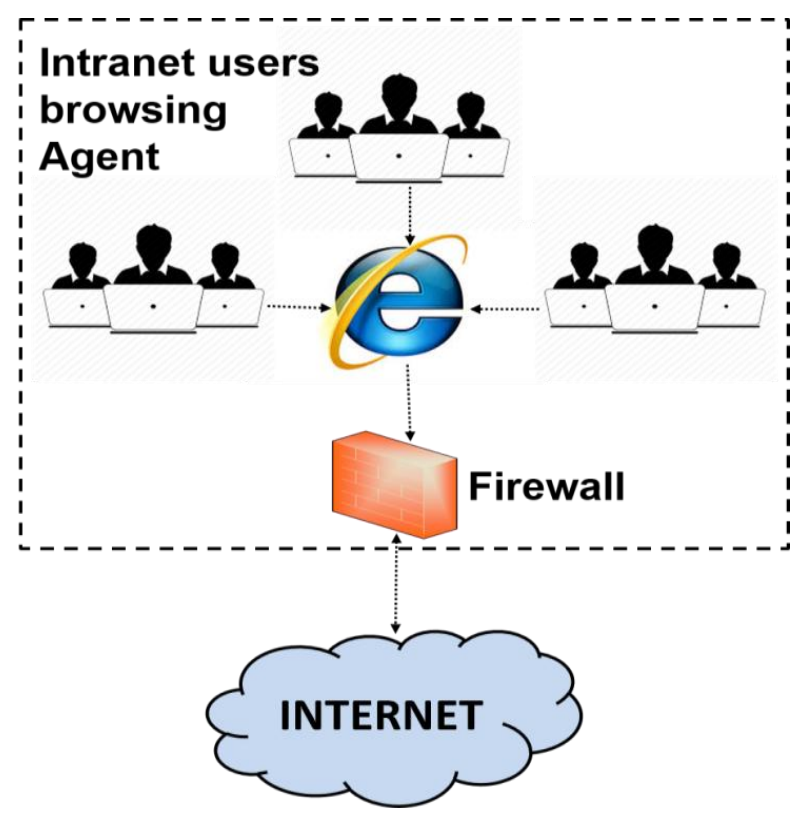

Fig 7: Architecture of Agent server in Intranet

After zip file upload into the Cloud server, there are many process at the server end to be followed which are given below:

- The copied zip file is now ready here to unzipped and open.

- As the zip file is password protected in the system, so after proper authentication the program extracts all the text file from the zip file.

- The unzip process results in the text files which was made after the data download from SAP. The code for unzip is given below:

ZipFile zf = ZipFile.Read(UploadPath + "CNData.zip");

zf.Password $=$

ConfigurationManager.AppSettings["WebServicePasswor

d"].ToString();

zf.ExtractAll(UploadPath,

ExtractExistingFileAction.OverwriteSilently);

\section{zf.Dispose();}

Now the text files are ready to be upload into the database (SQL Server) at the web server end. The process includes many sub-programs which transfer the data after proper manipulation and authentication of data into the SQL Server. The PL/SQL procedures and functions are included into the process and the failure at any step results in the rollback of whole the process so as to maintain the ACID properties of the transaction.

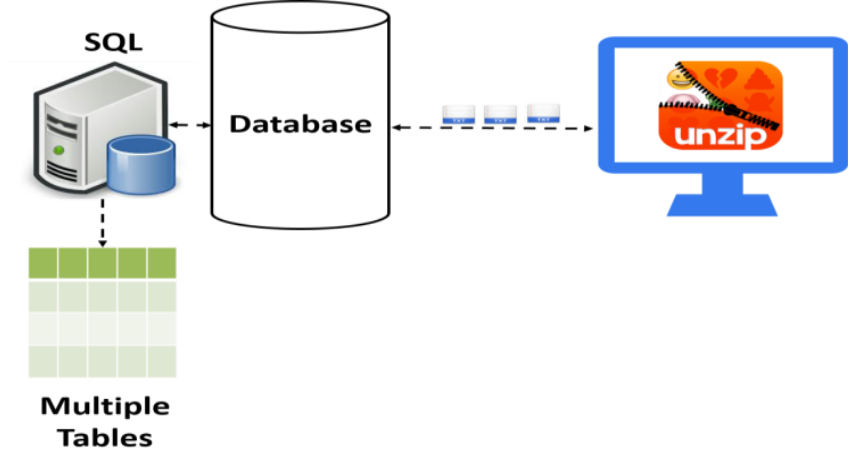

Fig 8: Processes in Cloud server end

The logs are associated with each of the process are recorded in the particular database table. There will be many tables after this process and the data will flow to the appropriate tables as per the rules are defined.

The part of the web services in this whole process uses different Web Methods as per the requirement of the system. The Web methods in C\# looks like as:

[WebMethod, SoapHeader("spAuthenticationHeader")] public string FireTrigger(string operationType, long createdBy)

\{

$$
\text { if (isValidUser()) }
$$

\{

return new

DBManager().FireTrigger(operationType, createdBy).ToString();

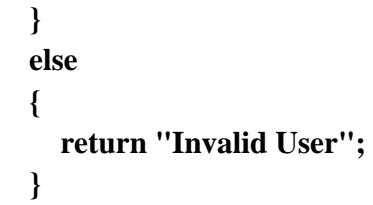

The users outside of the organization or protected network use internet to access the uploaded data from SAP and the process starts at the user's end as per whatever the requirement of the system is.

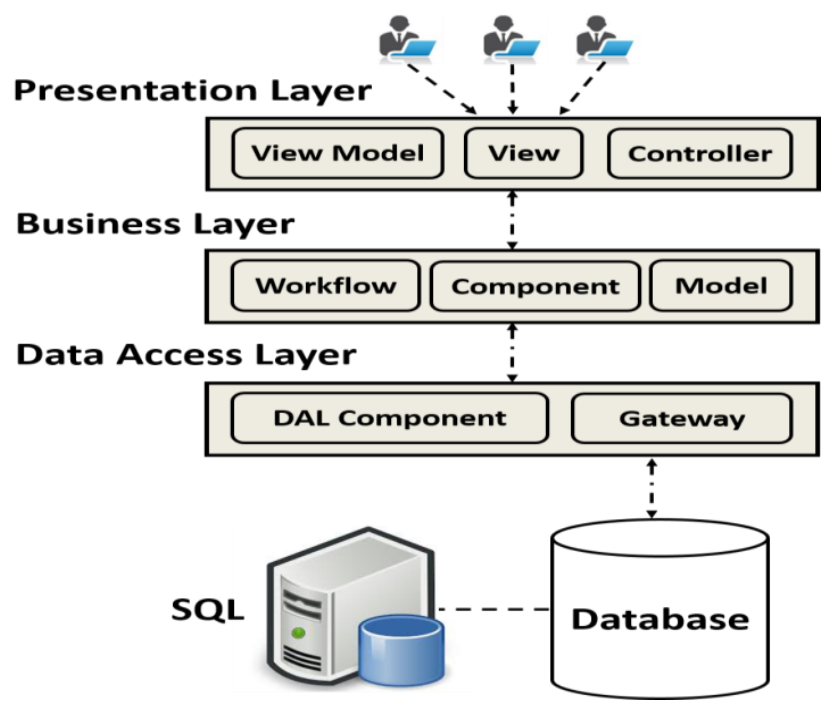

Fig 9: Layered architecture in Cloud web server 
The layered approach is shown in fig. 9 which consists of three layers namely Presentation, Business and data Access layers.

The data access by the user follow the three tier architecture in which each layer performs its own function and provide the appropriate data to the end users.

The user interface at the Cloud web server can be made as per the requirement including many more graphical user interface which is connected to cloud's database. The process then goes in reverse direction as there is need to transfer the data from cloud web server to SAP system.

\section{EXPERIMENTS AND RESULTS COMPARISON}

The authors have shown four no. of processes and time taken by these processes in seconds (table 1). The time taken is observed system processes and the data extraction from SAP, Agent processes on that data, Zipping, uploading into the cloud and Cloud processes.

Table 1. Records of processes with time

\begin{tabular}{|l|c|}
\hline Processes & Time(In ser \\
\hline RFC Communication & 15 \\
\hline Agent processes & 150 \\
\hline Upload Zip to Cloud & 53 \\
Cloud Process & 35
\end{tabular}

The time comparison pie is shown below which displays the four processes and time taken by these processes in percentage measurement (fig.10).

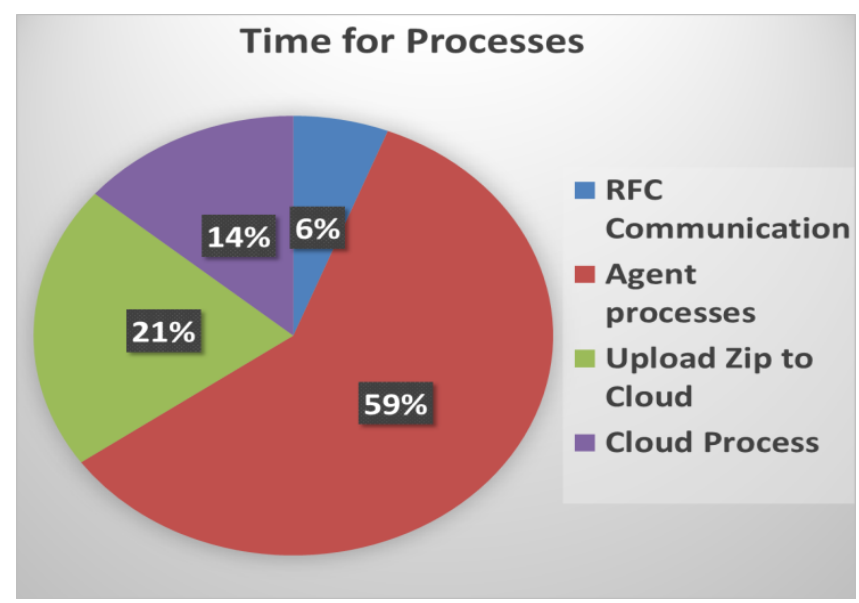

Fig 10: Process's time comparison pie

From above figure, it has been observed that Cloud processes is taking maximum time to execute.

The five processes are taken (table 2) from the set of processes and compared under two scenario (Centralized and Distributed firewall) (fig.11). These processes have same number of records in both the scenario.
Table 2. Records in data download process

\begin{tabular}{|l|l|l|l|l|l|}
\hline Processes & P1 & P2 & P3 & P4 & P5 \\
\hline Centralized(in sec) & $\mathbf{2 1}$ & $\mathbf{4 3}$ & $\mathbf{2 4}$ & $\mathbf{2 7}$ & $\mathbf{6 2}$ \\
\hline Distributed(in sec) & $\mathbf{1 3}$ & $\mathbf{3 7}$ & $\mathbf{2 0}$ & $\mathbf{2 3}$ & $\mathbf{5 0}$ \\
\hline
\end{tabular}

The data download under two scenario are different and shown by marker P1 to P5 with changing bars in fig. 11 .

\section{Download data from SAP}

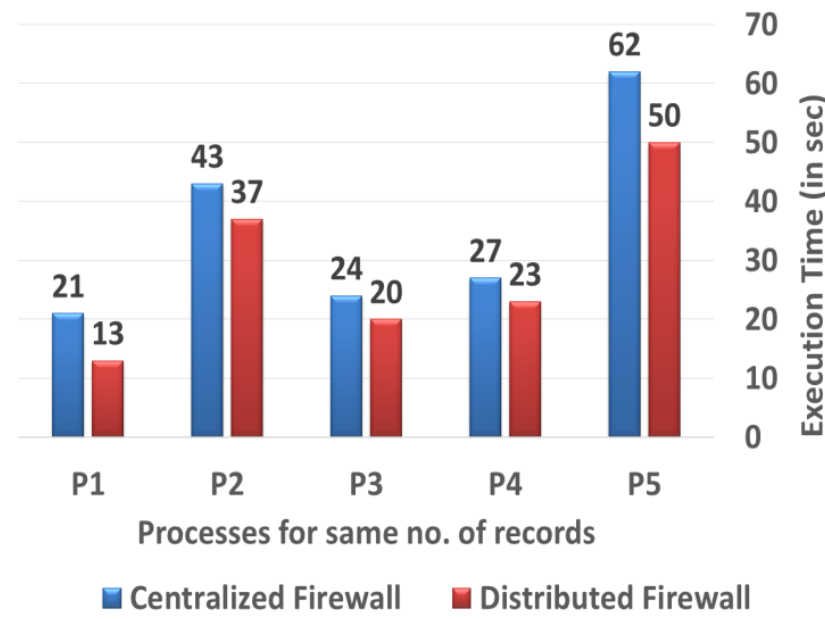

Fig 11: Comparison of data download under Centralized v/s Distributed Firewall

\section{FUTURE SCOPE}

The overall system interface is made possible to consume less time while execution using different proxy servers. Later on the system can be made more precisely to save more time and less resource consumption while communication. Sometimes the proxy server may not response properly at time. So there is need to find out more appropriate method to auto switch the proxy servers in less response time. In future when the no. of users will increase, the complexity also increases so as to maintain the neutrality and proper responsive system, the further research can be done. To make sure that the less no. of failures occurs while the communication and synchronization process, the research will goes on.

\section{CONCLUSION}

The authors firstly divided the whole communication process into four individual processes and then compared them in a pie in terms of time taken (in percentage) against no. of records in data download. So the authors concluded from this pie that the Agent process is taking more time as compared to other three processes and in future the time may be reduced by introducing new methods or technology. The reason behind this more time taken process is direct data download from SAP server to local SQL Server in Agent process.

Secondly, the authors compared the Agent processes (Download data from SAP) under two cases: use of Centralized and Distributed firewall into the internal organizational network. There are five processes chosen for the comparison in terms of time taken (in secs) having same no. of records in both the scenario. 


\section{ACKNOWLEDGMENTS}

This research paper is made possible through the help and support from everyone, including: parents, teachers, family, friends, and in essence, all sentient beings. Especially, please allow the authors to dedicate acknowledgment of gratitude toward the following significant advisors and contributors: The authors would like to thank University R\&D department and HOD, MMU for most support and encouragement. The contribution includes reading paper and offered invaluable detailed advices on grammar, organization, and the theme of the paper. Finally, the authors sincerely thank to parents, family, and friends, who provide the advice. The product of this research paper would not be possible without all of them.

\section{REFERENCES}

[1] Manish Kumar, Debasis Dwibedy, "Implementation of a Novel System for Cross Platform Communication of Diversified Applications over Network", International Journal of Computer Applications (0975 - 8887), Volume 128- No.9, October 2015.

[2] Dr. R. Seshadri, S. Venkateswarlu, "Web Application Security Model at Intranet Level Using HTTP", International Journal of Advanced Research in Computer Science and Software Engineering, Volume 5, Issue 7, July 2015.

[3] Surbhi Gupta, Puneet Bhalla, "Securing Wi-Fi Network via Proxy Servers", Research Inventy: International Journal of Engineering and Science, Vol.3, Issue 7 (August 2013).

[4] Vipin Saxena, Ajay Pratap, "Representation of ObjectOriented Database for the Development of Web Based Application Using Db4o", Journal of Software Engineering and Applications, 2012, 5, 687-694.

[5] Xiangjun Li, Liang Huang, Zhenrong Lin, ZiLong Sai, "Research of Routing System which applied in ASP.NET MVC Application", Proceedings of the Second Symposium International Computer Science and Computational Technology(ISCSCT '09).

[6] Minakshi, Happy Sharma, "Web Technology: Http, Web Server \& Web Services", International Journal of Research (IJR) Vol-1, Issue-10 November 2014.

[7] Chu-Hsing Lin, Jung-Chun Liu, Chien-Ting Kuo, MeiChun Chou, Tsung-Che Yang, "Safeguard Intranet Using Embedded and Distributed Firewall System",
International Journal of Future Generation Communication and Networking, Vol. 2, No. 1, March, 2009.

[8] Kalpana Johari, Arvinder Kaur, "Some Interoperability Issues In The Designing Of Web Services: Case Study On Credit Card", International Journal on Web Service Computing (IJWSC), Vol.4, No.4, December 2013.

[9] Ya-Ling Wang, Ying-Chieh Chang, Quincy Wu, "Intelligent Proxy Server in TANet/TWAREN", Proceedings of the Asia Pacific Advanced Network.

[10] Boon-Wee Low, Boon-Yaik Ooi, Chee-Siang Wong, "Exploration on Scalability of Database Bulk Insertion with Multi-threading", International Journal on New Computer Architectures and Their Applications (IJNCAA) 1(3): 553-564, The Society of Digital Information and Wireless Communications, 2011 (ISSN: 2220-9085).

[11] Kanagaraj.S, Dr.Sunitha Abburu, "Converting Relational Database into Xml Document", IJCSI International Journal of Computer Science Issues, Vol. 9, Issue 2, No 1, March 2012.

[12] Ahmed Mohamed Lamey, Dr.Hamdy M. Mousa, Dr.Arabi keshk, "Improve Performance of Enterprise Information Sap Portal", International Journal of Computer Applications (0975 - 8887), Volume 37No.1, January 2012.

[13] Maitham Ali Naji, "Implementation of Converting Text File to Data Access Table by Using Multi-Keys", International Journal Of Scientific \& Engineering Research, Volume 4, Issue 12, December-2013.

[14] Dr.S.S.Riaz Ahamed, "Moving Toward Network Security And Firewalls For Protecting And Preserving Private Resources On The Internet", Journal of Theoretical and Applied Information Technology, (20052008).

[15] Anupam Das, Avick Kumar Dey, Baishakhi Karmakar, Anirban Das, "A Cutting Edge Approach in Bridging between Microsoft .NET Framework and SAP R/3", International Symposium on Devices MEMS, Intelligent Systems \& Communication (ISDMISC) 2011, Proceedings published by International Journal of Computer Applications (IJCA). 